\title{
Measuring Microgel Swell Ratio by cryo-SEM
}

\author{
Jing Liang ${ }^{\text {a }}$, Feiyue Teng ${ }^{\text {a }}$, Tseng-Ming Chou ${ }^{\text {a, }}$, Matthew Libera ${ }^{\text {a* }}$
}

a. Department of Chemical Engineering \& Materials Science, Stevens Institute of Technology, Hoboken, NJ USA

b. Laboratory for Multiscale Imaging, Stevens Institute of Technology, Hoboken, NJ USA 


\section{Abstract:}

The swell ratio is a key parameter characterizing the structure and properties of a hydrogel. In macroscopic gels, the swell ratio can be determined by straightforward measurements of the gel weight in the dry and hydrated states. However, measuring the swell ratio characteristic of microgels (gel particles with dimensions of $0.1-100 \mu \mathrm{m}$ ) is substantially more challenging because of their small size and polydispersity. We use cryo-scanning electron microscopy (cryoSEM) to measure microgel diameter both in the frozen-hydrated and fully dry states using $\mathrm{pH}$ responsive poly(ethylene glycol)-co-acrylic acid microgels. The volume swell ratios characteristic of the various microgels are essentially the same as those measured from otherwise-identical macroscopic gels. Hence we can conclude that, at least in this case where the macroscopic gel and microgel synthesis methods are similar, the simple measurement of a macroscopic swell ratio provides a reasonable approximation to the microgel swell ratio. 
Perhaps the most significant quantity characterizing a hydrogel is its swell ratio. The swell ratio is a macroscopic manifestation of the gel mesh size and crosslink density. These molecular-level parameters in turn control mechanical and transport properties critical to applications such as tissue engineering and drug delivery. The relationship between swell ratio and mesh size was initially developed by Flory and Rehner [1, 2]. The volume swell ratio, Q, is given as:

$$
Q=\frac{V_{s}}{V_{d}}
$$

where $V_{s}$ is the volume of swollen network at equilibrium and $V_{d}$ is the volume of unswollen (dry) network. For a macroscopic hydrogel, determining the volume swell ratio is straightforward from measurements of the hydrogel weight in the dry, $m_{d}$, and water-swollen, $\mathrm{m}_{\mathrm{s}}$, states:

$$
\mathrm{Q}=1+\frac{\rho_{p}}{\rho_{s}}\left(\frac{m_{s}}{m_{d}}-1\right)
$$

where the polymer and solvent densities are $\rho_{\mathrm{p}}$ and $\rho_{\mathrm{s}}$, respectively. The gel mesh size can then be calculated from the swell ratio using the Flory-Rehner theory, and this approach has been applied to a number of neutral polymer gel systems such as PEG and poly(vinyl alcohol) [3, 4]. Peppas et al. [5] further modified the Flory-Rehner theory to address polyelectrolyte gel systems where issues of charge, $\mathrm{pH}$, and ionic strength can contribute substantially to the gel-swelling behavior. 
Microgels are gel particles with sizes in the range of 0.1-100 $\mu \mathrm{m}$ [6]. They are being

1
used increasingly in a variety of colloid, drug-delivery, and surface-modification applications [710], which, just as in their macroscopic gel counterparts, require control of the mesh size. However, in contrast to a macroscopic gel whose dimensions enable reasonably easy and accurate measurements of the dry and swollen weights, the swelling behavior of microgels is much more difficult to characterize. Dynamic light scattering, for example, only provides the intensity-averaged hydrodynamic diameter and does so just for relatively small (sub-micron sized) microgels. Weight measurements can be made using samples containing a large number of microgels to mimic a macroscopic sample, but fully removing interstitial water between microgels is difficult and consequently can lead to errors in measuring the swollen weight.

Here we ask the simple question of how well a measurement of swell ratio from a macroscopic gel compares to that of a microgel synthesized under identical conditions composition, solvent, UV exposure, etc. - except for the finely divided nature of the microgel. We use cryo-SEM to make direct measurements of microgel size in the frozen-hydrated (swollen) and dehydrated (dry) states, and, at least in the particular case involving poly(ethylene glycol)-co-acrylic acid (PEG-co-AA) gels and microgels, we find excellent agreement between the swell ratio characteristic of the macroscopic gels and the microgels.

PEG-co-AA gels and microgels were synthesized by free radical polymerization. The microgels were formed by membrane emulsification using a precursor solution of $2 \mathrm{~mL}$ PEG diacrylate (PEGDA; $M_{n}=575 \mathrm{~g} / \mathrm{mol}$; Sigma), $200 \mu \mathrm{L}$ acrylic acid (Sigma), and $100 \mu \mathrm{L}$ photo- 


7

initiator (Darocur 1173; Sigma) all dissolved in $10 \mathrm{~mL}$ dichloromethane. The precursor solution was dispersed as microscopic droplets in $100 \mathrm{~mL}$ deionized water (DI) by forcing the precursor solution through a Shirasu Porous Glass (SPG) membrane [11]. The resulting emulsion was exposed to $450 \mathrm{~W}$ UV light for 15 minutes. After solvent removal, the microgels were washed by repeated centrifugation and re-suspension in DI water. Macroscopic hydrogels with dimensions on the order of $1 \mathrm{~cm}$ were prepared by exposing $1 \mathrm{~mL}$ of precursor solution to $450 \mathrm{~W} \mathrm{UV}$ light for 15 minutes. To better mimic the microgel synthesis conditions as well as prevent dichloromethane evaporation, the precursor solution was covered by $10 \mathrm{~mL}$ of DI water during photo-polymerization. The resulting gels were soaked in DI water and heated to $50{ }^{\circ} \mathrm{C}$ while dichloromethane was removed by vacuum. The gels were then soaked for 3 days in a large bath of DI water. The swollen weight was obtained after soaking the sample in either $\mathrm{pH} 3$ or $\mathrm{pH} 9$ in $0.01 \mathrm{M}$ phosphate buffer for 48 hours. The dry weight of the macroscopic gels was obtained by dehydrating the gels at $50{ }^{\circ} \mathrm{C}$ for 48 hours. Prior to the hydrated weight measurement, excess surface buffer was removed by gently patting the gel surfaces with filter paper.

8

Cryo-SEM used frozen-hydrated samples were prepared by high-pressure freezing (HPF). $5 \mu \mathrm{L}$ of microgels suspended in $\mathrm{pH} 3$ or $\mathrm{pH} 9$ buffer $(0.01 \mathrm{M}$ phosphate buffer $)$ were frozen using a Leica EM HPM100 HPF system. High-pressure freezing promotes water amorphization while freezing by plunging in liquid nitrogen (LN2) at atmosphere pressure tends to create crystalline ice, which can induce artifactual specimen structure $[12,13]$. HPF microgel samples were cryo-fractured and cryo-transferred to a Zeiss Auriga Cross-Beam FIB-SEM equipped with a Leica VCT-100 cryo-transfer system. Frozen-hydrated samples were imaged using $1 \mathrm{keV}$ electrons at a temperature of $-135^{\circ} \mathrm{C}$ and a vacuum of $2 \times 10^{-6}$ mbar. Dried samples were imaged 
at room temperature. To remove water from the frozen-hydrated microgel suspensions, the

107 sample temperature was raised to $-95{ }^{\circ} \mathrm{C}$ to sublimate water into the microscope vacuum. We used sublimation times of $10 \mathrm{~min}$ and $40 \mathrm{~min}$ to remove relatively small amounts (microns) of

109 water from the sample surface. Samples were

110 re-cooled to $-135{ }^{\circ} \mathrm{C}$ for cryo-SEM

111 observation Alternatively, fully dehydrated

112 specimens were produced by warming the

113 specimen to $-95{ }^{\circ} \mathrm{C}$ for several hours until the

114 cryo-stage's LN2 supply was exhausted,

115 whereupon the specimen slowly warmed to

116 room temperature over a period of

117 approximately $12 \mathrm{~h}$. The water content of the

118 microgels after these three sublimation

119 processes was followed by monitoring the

120 intensity ratio of carbon and oxygen X-rays

121 collected by energy dispersive X-ray

122 spectroscopy (Oxford Max-80 SDD EDS

123 system).

Figure 1 presents typical SEM images

126 showing the microgels after each of the three

127 different sublimation treatments. Importantly, the microgels appear smooth and spherical.
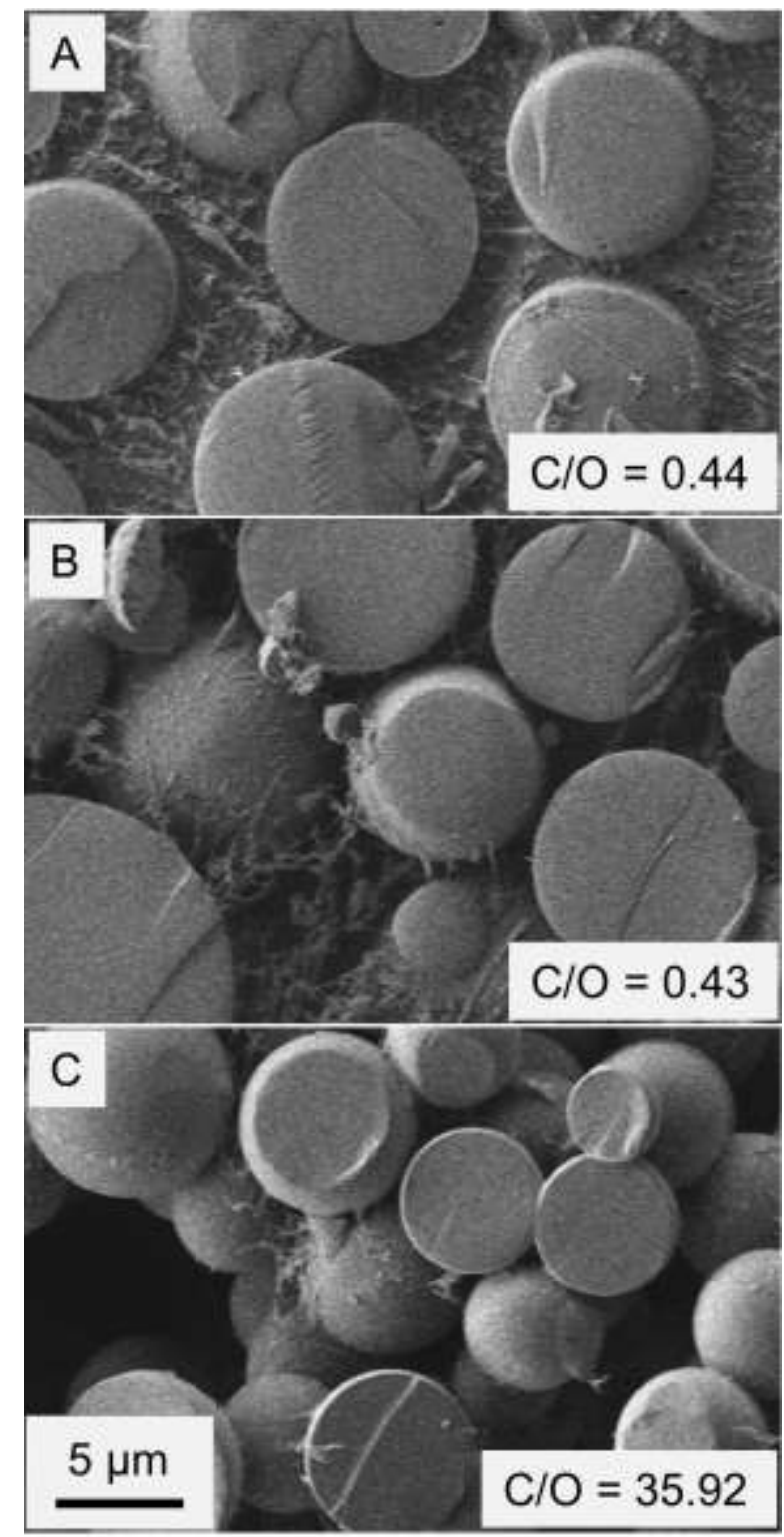

Figure 1 - Cryo-SEM (A, B) and room-temperature SEM (C) images of frozen-hydrated microgel suspensions $(\mathrm{pH} 3)$ after sublimation treatments of: (A) $10 \mathrm{~min}$; (B) $40 \mathrm{~min}$; and (C) $12 \mathrm{~h}$. The insets indicate the ratios of carbon and oxygen X-ray intensities. 
10 min of sublimation removes $\sim 1 \mu \mathrm{m}$ of water, leaving the microgels significantly embedded in ice (Fig. 1A). The microgel C/O X-ray intensity ratio (Fig. 1A inset) is 0.44 . Note that this ratio does not directly correspond to the composition ratio, and we did not pursue the various corrections and calibrations required to make this conversion. Furthermore, $1 \mathrm{keV}$ electrons travel only about $100 \mathrm{~nm}$ into the specimen. X-ray generation is consequently localized primarily to the near-surface region of the individual microgels analyzed. After the $40 \mathrm{~min}$ sublimation treatment (Fig. 1B) the topmost layer of microgels in the frozen-hydrated is almost entirely free from surrounding ice. Nevertheless, the microgel C/O X-ray intensity ratio (Fig. 1B inset) under the $40 \mathrm{~min}$ sublimation is essentially the same $(0.43)$ as that after $10 \mathrm{~min}$. This observation indicates that water is preferentially sublimated from the ice surrounding the individual microgels, while bound water remains within the exposed microgels. However, even this bound water is removed when the microgels are subjected to the extended sublimation process followed by warming to room temperature under the high vacuum of the microscope (Fig. 1C). Under this condition, the $\mathrm{C} / \mathrm{O} \mathrm{X}$-ray intensity ratio increases to almost 36 . We measured a similar ratio from thick microgel specimens, which were dried for 3 days at $50{ }^{\circ} \mathrm{C}$ and then analyzed under identical SEM imaging and EDS X-ray collection conditions. We can thus be confident that the $12 \mathrm{~h}$ sublimation treatment produces fully dried microgels. Importantly, the dried microgels maintain their spherical structure and smooth surface, suggesting that the de-swelling process is isotropic. specimens subjected to the partial and full sublimation treatments such as those in Figures 1B and $1 \mathrm{C}$, respectively. Since the freeze-fracture process produces a specimen surface presenting 
152 fractured microgels, some of which have an ( 4.5).

169 dried microgel diameter, respectively:

exposed face diameter less than that of an exact hemisphere, care was taken to measure the diameter of a fractured microgel only when its maximum diameter was visible. Figure 2 presents size-distribution histograms, together with a Gaussian fit to each data set, for dry and frozen-hydrated microgels that had been swollen either in $\mathrm{pH} 3$ or $\mathrm{pH} 9$ buffer. Each dataset on these histograms represents measurements made from over 100 different microgels. The frozen-hydrated microgels swollen in $\mathrm{pH} 9$ buffer have swell ratios larger than those swollen in $\mathrm{pH} 3$ buffer because of the increased swelling at $\mathrm{pH}$ values higher than the pKa of AA carboxyl group 174
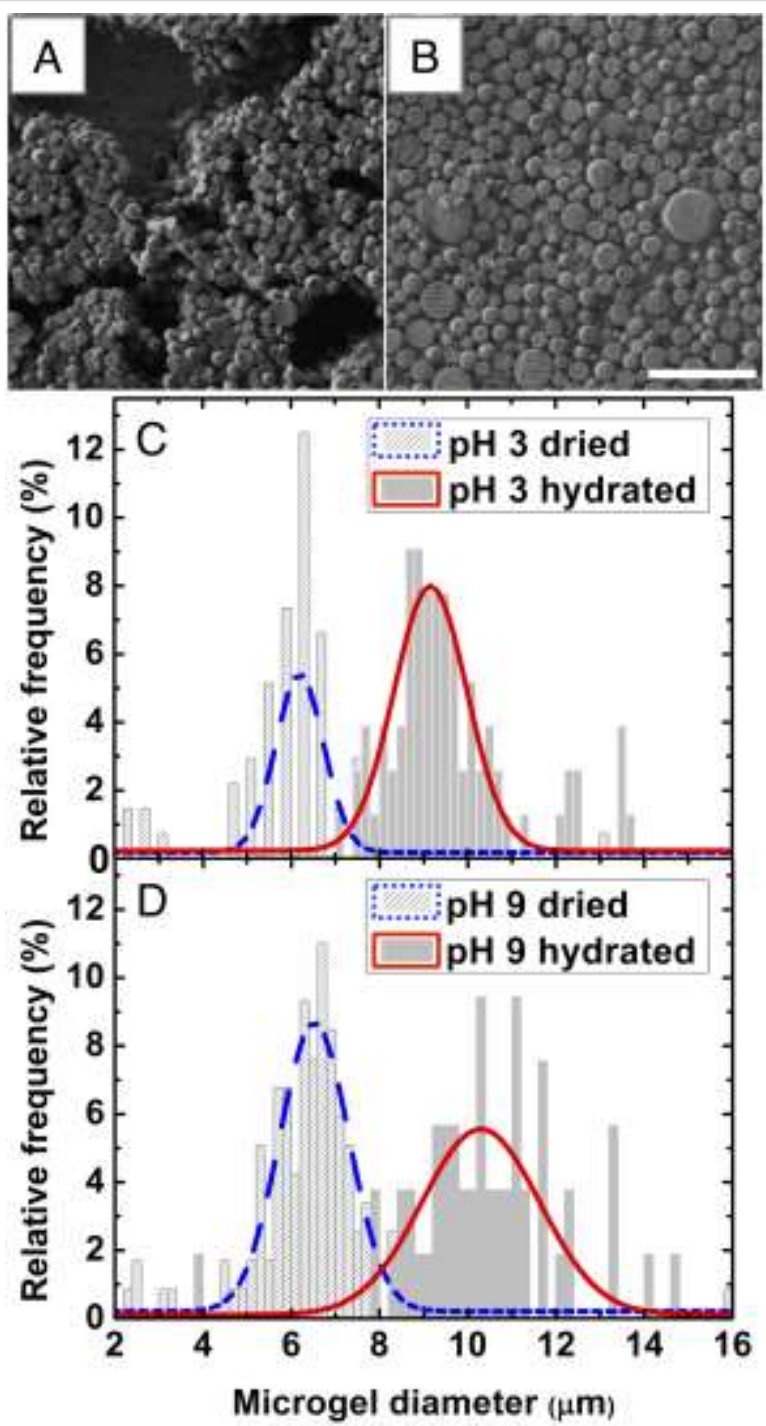

Figure 2 - (A) Room-temperature SEM image of dried microgels. (B) Cryo-SEM image of frozen-hydrated microgels after a $40 \mathrm{~min}$ sublimation treatment. The scale bar represents $50 \mu \mathrm{m}$ for both A and B. Sizedistribution histograms of dried (12 h sublimation) and frozen-hydrated (40 min sublimation) at: (C) $\mathrm{pH}$ 3; and (D) pH 9. The solid and dashed lines represent Gaussian fits to each data set (>100 measurements per data set).

calculate the volume swell ratio using equation (3), where $d_{s}$ and $d_{d}$ are the average hydrated and 
176 Measurements of macroscopic gel weights in

177 the dry $\left(\mathrm{m}_{\mathrm{s}}\right)$ and hydrated $\left(\mathrm{m}_{\mathrm{d}}\right)$ conditions

178 were used to calculate the volume swell ratio

179 using equation (2), assuming the PEG-co-AA

180 copolymer density $\left(\rho_{\mathrm{p}}\right)$ is $1.12 \mathrm{~g} / \mathrm{cm}^{3}$ and

181 water density $\left(\rho_{\mathrm{s}}\right)$ is $1 \mathrm{~g} / \mathrm{cm}^{3}$. The results are

182 summarized by Table 1 . The swell ratios
Table 1: Volume swell ratios

\begin{tabular}{|c|c|c|}
\hline & pH 3 & pH 9 \\
\hline Microgel $^{\text {a }}$ & $3.3 \pm 1.3$ & $4.0 \pm 2.1$ \\
\hline Macroscopic gel ${ }^{\mathrm{b}}$ & $3.4 \pm 0.1$ & $3.9 \pm 0.1$ \\
\hline \multicolumn{3}{|c|}{$\begin{array}{l}\text { a } \text { The microgel average diameter } \pm \text { standard } \\
\text { deviation are derived from Gaussian fits in } \\
\text { Figure. } 2 \text {. } \\
\text { b The average } \pm \text { standard deviation are each } \\
\text { derived from weight measurements of } 5 \text { gels. }\end{array}$} \\
\hline
\end{tabular}

183 characterizing the bulk gels are almost identical to those of the microgels.

184

185

186

187

188

189

190

191

192

193

194

195

196

197

Following reports in the literature [14-16], we also measured the hydrated microgel swell ratio by analyzing the weight of microgel pellets after centrifugation. As we would do with a hydrated bulk gel, we removed excess water by gently patting the pellets with filter paper. We used 5 different pellets for each $\mathrm{pH}$ condition, and, after measuring the hydrated weight, we then dried the pellets at $50{ }^{\circ} \mathrm{C}$ for 2 days. Using this method together with equation (2), we measured microgel volume swell ratios of $6.2 \pm 0.5$ at $\mathrm{pH} 3$ and $16.3 \pm 0.7$ at $\mathrm{pH} 9$. These values are substantially higher than those reported in Table 1. We attribute those differences to excess interstitial water in the hydrated microgel pellet that is difficult to remove because of the substantially higher surface area associated with the microgels relative to a macroscopic gel.

To further confirm the accuracy of the cryo-SEM measurements, we followed a set of the same microgels from the frozen-hydrated state to the fully dehydrated state. The set of microgels is shown in Fig. 3. These were hydrated in $\mathrm{pH} 9$ buffer. Unlike the statistical approach reported 
in Figure 2, image data such as that in Figure 3 enable direct measurements of microgel size and thus determine the swell ratio characteristic of individual microgels. The microgel sizes after the $10 \mathrm{~min}$ and $40 \mathrm{~min}$ sublimation treatments are again identical. They undergo isotropic deswelling with the 12

h sublimation/warming process, each ending with a substantially smaller dry size. We again used equation (3) to determine the microgel-specific $\mathrm{pH} 9$ swell ratio. By analyzing the cross-sectional diameters of ten different microgels in the hydrated and dry states, the volume swell ratio obtained was 4.1 \pm 0.5 . This value is essentially the same as that reported in Table 1. SEM to make very specific and accurate
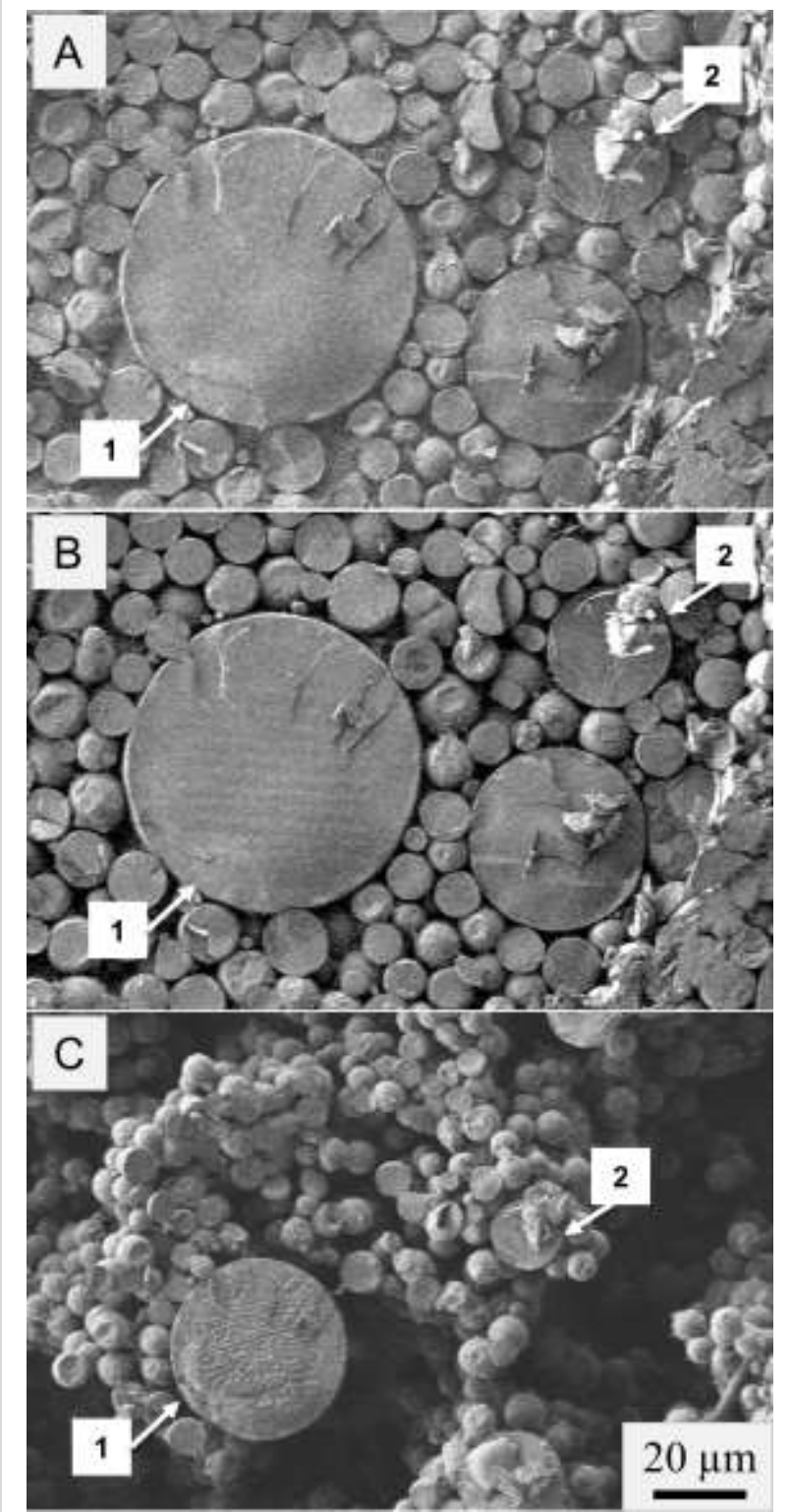

Figure 3 - Cryo-SEM (A, B) and room-temperature SEM (C) images of the same set of microgels after sublimation for: (A) $10 \mathrm{~min}$; (B) $40 \mathrm{~min}$; and (C) $12 \mathrm{~h}$. The same two microgels, labeled 1 and 2 , are marked in each image.

measurements of microgel swell ratio in the PEG-co-AA system. These ratios compare extremely well with those measured from macroscopic hydrogels synthesized from the same precursor solution but without the membrane-emulsification process. In this particular case, we can thus conclude that making the far simpler measurement of a swell ratio from a macroscopic 
221 gel can provide an accurate characterization of the network properties of both the macroscopic 222 gel and microgel. Extrapolating beyond this specific case, the relationship between macroscopic 223 gels and microgels is likely to be maintained for similar compositions and similar microgel 224 synthesis methods. Hence we can conclude that, at least in this case where the macroscopic gel 225 and microgel synthesis methods are similar, the simple measurement of a macroscopic swell 226 ratio provides a reasonable approximation to the microgel swell ratio.

\section{Acknowledgments:}

This work has been supported by the United States National Science Foundation (grant

230 \#DMR-1608406) and used microscopy resources supported by the National Science Foundation 231 (grants \#DMR-1428296 and DMR-0922522) and the Stevens Institute of Technology. 


\section{References:}

[1] P.J. Flory, J. Rehner Jr, Statistical mechanics of cross-linked polymer networks I. Rubberlike elasticity, The Journal of Chemical Physics 11 (1943) 512-520.

[2] P.J. Flory, Statistical mechanics of swelling of network structures, The Journal of Chemical Physics 18 (1950) $108-111$.

[3] P. Martens, T. Holland, K.S. Anseth, Synthesis and characterization of degradable hydrogels formed from acrylate modified poly (vinyl alcohol) macromers, Polymer 43 (2002) 6093-6100.

[4] V. Truong, I. Blakey, A.K. Whittaker, Hydrophilic and amphiphilic polyethylene glycol-based hydrogels with tunable degradability prepared by "click" chemistry, Biomacromolecules 13 (2012) 4012-4021.

[5] L. Brannon-Peppas, N.A. Peppas, Equilibrium swelling behavior of pH-sensitive hydrogels, Chemical Engineering Science 46 (1991) 715-722.

[6] V. Jarm, Compendium of Polymer Terminology and Nomenclature, Chemical and Biochemical Engineering Quarterly 24 (2010) 249-252.

[7] L. Nyström, M. Malmsten, Surface-bound microgels-From physicochemical properties to biomedical applications, Advances in Colloid and Interface Science 238 (2016) 88-104.

[8] R. Pelton, Temperature-sensitive aqueous microgels, Advances in Colloid and Interface Science 85 (2000) 1-33.

[9] B. Xue, V. Kozlovskaya, E. Kharlampieva, Shaped stimuli-responsive hydrogel particles: syntheses, properties and biological responses, Journal of Materials Chemistry B 5 (2017) 9-35.

[10] B.R. Saunders, N. Laajam, E. Daly, S. Teow, X. Hu, R. Stepto, Microgels: From responsive polymer colloids to biomaterials, Advances in Colloid and Interface Science 147 (2009) 251-262.

[11] S. Omi, Preparation of monodisperse microspheres using the Shirasu porous glass emulsification technique, Colloids and Surfaces A: Physicochemical and Engineering Aspects 109 (1996) 97-107.

[12] R. Aston, K. Sewell, T. Klein, G. Lawrie, L. Grondahl, Evaluation of the impact of freezing preparation techniques on the characterisation of alginate hydrogels by cryo-SEM, European Polymer Journal 82 (2016) 1-15.

[13] Y. Wu, J. Liang, K. Rensing, T.-M. Chou, M. Libera, Extracellular Matrix Reorganization during Cryo Preparation for Scanning Electron Microscope Imaging of Staphylococcus aureus Biofilms, Microscopy and Microanalysis 20 (2014) 1348-1355.

[14] M.B. Sobhanimatin, S. Pourmahdian, Study on the inverse emulsion copolymerization of microgels based on acrylamide/2-acrylamido-2-methylpropane sulfonic acid, Iranian Polymer Journal 25 (2016) 405-413.

[15] K. Hemmati, A. Masoumi, M. Ghaemy, pH responsive tragacanth gum and poly(methyl methacrylate-comaleic anhydride)-g-poly(caprolactone) conetwork microgel for in vitro quercetin release, Polymer 59 (2015) 49-56.

[16] J. Stukel, S. Thompson, L. Simon, R. Willits, Polyethlyene glycol microgels to deliver bioactive nerve growth factor, Journal of Biomedical Materials Research Part A 103 (2015) 604-613. 